\title{
Square functions and the Hamming cube: Duality
}

\author{
Paata Ivanisvili* $\quad$ Fedor Nazarov $^{\dagger} \quad$ Alexander Volberg ${ }^{\ddagger}$
}

Received 6 July 2017; Published 19 January 2018

\begin{abstract}
For $1<p \leq 2$, any $n \geq 1$ and any $f:\{-1,1\}^{n} \rightarrow \mathbb{R}$, we obtain $\left(\mathbb{E}|\nabla f|^{p}\right)^{1 / p} \geq$ $c(p)\left(\mathbb{E}|f|^{p}-|\mathbb{E} f|^{p}\right)^{1 / p}$ where $c(p)$ is the smallest positive zero of the confluent hypergeometric function ${ }_{1} F_{1}\left(\frac{p}{2(1-p)}, \frac{1}{2}, \frac{x^{2}}{2}\right)$. Our approach is based on a certain duality between the classical square function estimates on the Euclidean space and the gradient estimates on the Hamming cube.
\end{abstract}

Key words and phrases: Square function, Hamming cube, Duality

\section{Main result}

Consider the Hamming cube $\{-1,1\}^{n}$ of an arbitrary dimension $n \geq 1$. For any $f:\{-1,1\}^{n} \rightarrow \mathbb{R}$ define the discrete partial derivative $\partial_{j} f(x)$ as follows

$$
\partial_{j} f(x)=\frac{f(x)-f\left(S_{j}(x)\right)}{2}, \quad x=\left(x_{1}, \ldots, x_{n}\right) \in\{-1,1\}^{n},
$$

where $S_{j}(x)$ is obtained from $x$ by changing the sign of $j$ 'th coordinate of $x$. Set $\nabla f(x):=\left(\partial_{1} f(x), \ldots, \partial_{n} f(x)\right)$, and we define the norm of the discrete gradient

$$
|\nabla f|^{2}(x):=\sum_{j=1}^{n}\left(\partial_{j} f(x)\right)^{2}=\sum_{y \sim x}\left(\frac{f(x)-f(y)}{2}\right)^{2},
$$

where the summation in the last term runs over all neighbor vertices of $x$ in $\{-1,1\}^{n}$. Set

$$
\mathbb{E} f=\frac{1}{2^{n}} \sum_{x \in\{-1,1\}^{n}} f(x) .
$$

*This paper is based upon work supported by the National Science Foundation under Grant No. DMS-1440140 while two of the authors were in residence at the Mathematical Sciences Research Institute in Berkeley, California, during the Spring and Fall 2017 semester.

${ }^{\dagger}$ Supported by NSF DMS-0800243

$\ddagger$ Supported by NSF DMS-1600065 
Theorem 1.1. For any $1<p \leq 2, n \geq 1$, and any $f:\{-1,1\}^{n} \rightarrow \mathbb{R}$ we have

$$
s_{p^{\prime}}\left(\mathbb{E}|f|^{p}-|\mathbb{E} f|^{p}\right)^{1 / p} \leq\left(\mathbb{E}|\nabla f|^{p}\right)^{1 / p} .
$$

Here $p^{\prime}=\frac{p}{p-1}$ is the conjugate exponent of $p$, and by $s_{q}$ we denote the smallest positive zero of the confluent hypergeometric function ${ }_{1} F_{1}\left(-\frac{q}{2}, \frac{1}{2}, \frac{x^{2}}{2}\right)$ (see (6) for the definition).

In Lemma A.2 we obtain a lower bound $s_{p^{\prime}} \geq \sqrt{2 / p^{\prime}}$ for $1<p \leq 2$ which is precise when $p \rightarrow 2$. If $p^{\prime}=2 k$ for $k \in \mathbb{N}$, then $s_{p^{\prime}}$ becomes the smallest positive zero of the Hermite polynomial $H_{2 k}(x)$ where

$$
H_{m}(x)=\int_{\mathbb{R}}(x+i y)^{m} \frac{e^{-y^{2} / 2}}{\sqrt{2 \pi}} d y .
$$

The constant $s_{p^{\prime}}$ in (1) is larger then all previously known bounds [15, 2] when $p$ is in a neighborhood of 2 , say $p \in(1.26,2)$. For example, the estimate (1) improves the Naor-Schechtman bound [15] for the class of real valued functions for all $1<p<2$. Indeed, it follows from an application of Khinchin inequality with the sharp constant and (1) that we have the following corollary

Corollary 1.2. For any $1<p \leq 2, n \geq 1$, and any $f:\{-1,1\}^{n} \rightarrow \mathbb{R}$ we have

$$
s_{p^{\prime}}^{p} 2^{\frac{p-2}{2}} \min \left(1, \frac{\Gamma((p+1) / 2)}{\Gamma(3 / 2)}\right) \mathbb{E}|f-\mathbb{E} f|^{p} \leq \mathbb{E}_{x} \mathbb{E}_{x^{\prime}}\left|\sum_{j=1}^{n} x_{j}^{\prime} \partial_{j} f(x)\right|^{p},
$$

where $\mathbb{E}_{x}$ and $\mathbb{E}_{x^{\prime}}$ average in variables $x$ and $x^{\prime}=\left(x_{1}^{\prime}, \ldots, x_{n}^{\prime}\right) \in\{-1,1\}^{n}$ correspondingly.

We will see in Proposition 3.4 that

$$
s_{p^{\prime}}^{p} 2^{\frac{p-2}{2}} \min \left(1, \frac{\Gamma((p+1) / 2)}{\Gamma(3 / 2)}\right)>(p-1)^{p} \quad \text { for } \quad 1<p<2 .
$$

The latter implies that the estimate (2) improves the bound of Naor-Schechtman for $1<p<2$ in the case of real valued functions (see Theorem 1 in [15] where $\beta_{p}(\mathbb{R})=1 /(p-1)$ ).

On the other hand $s_{p^{\prime}}$ degenerates to 0 when $p \rightarrow 1+$ which should not be the case for the best possible constant by a result of Talagrand (see Section 3.5). For this endpoint case, when $p$ is close to 1 , the result of Ben-Efraim-Lust-Piquard [2] gives the better bounds

$$
\frac{2}{\pi}\left(\mathbb{E}|f-\mathbb{E} f|^{p}\right)^{1 / p} \leq\left(\mathbb{E}|\nabla f|^{p}\right)^{1 / p} \quad 1 \leq p \leq 2,
$$

and when $p=1$ it is widely believed that the sharp constant in the left hand side of (3) should be $\sqrt{2 / \pi}$ instead of $2 / \pi$ (see Section 3.5 for more details).

We think that the main contribution of the current paper is not just Theorem 1.1 that we obtain but rather a new duality approach that we develop between two different classes of extremal problems: square function estimates on the interval $[0,1]$ and gradient estimates on the Hamming cube, and Theorem 1.1 should be considered as an example. Roughly speaking one can take a valid estimate for a square function, dualize it by a certain double Legendre transform, and one can write its corresponding dual estimate on the Hamming cube and vice versa. To illustrate another example of our duality approach, in Section 3.4 we present a short proof of the following theorem which improves a well-known inequality of Beckner 
Theorem 1.3 (see [11]). For any $n \geq 1$, and any $f:\{-1,1\}^{n} \rightarrow \mathbb{R}$ we have

$$
\mathbb{E} \mathfrak{R}(f+i|\nabla f|)^{3 / 2} \leq(\mathfrak{R} f)^{3 / 2},
$$

where $\Re$ denotes the real part, and $z^{3 / 2}$ is understood in the sense of principal brunch in the upper half-plane.

Going back to Theorem 1.1, it will be explained later that $s_{p^{\prime}}$ in a "dual" sense coincides with the sharp constant found by B. Davis in the $L^{q}$ norm estimates

$$
\begin{aligned}
& s_{q}\left\|T^{1 / 2}\right\|_{q} \leq\left\|B_{T}\right\|_{q}, \quad q \geq 2, \quad\left\|T^{1 / 2}\right\|_{q}<\infty ; \\
& \left\|B_{T}\right\|_{p} \leq s_{p}\left\|T^{1 / 2}\right\|_{p}, \quad 0<p \leq 2 .
\end{aligned}
$$

Here $B_{t}$ is the standard Brownian motion starting at zero, and $T$ is any stopping time. It was explained in [8] that the same sharp estimates (4) and (5) hold with $B_{T}$ replaced by an integrable function $g$ on $[0,1]$ with mean zero, and $T^{1 / 2}$ replaced by the dyadic square function of $g$.

We notice the essential difference between the Davis estimates (4), (5) and (1) that for a given power $p, 1<p \leq 2$, we need the "dual" constant $s_{p^{\prime}}=s_{\frac{p}{p-1}}$ in the theorem. Besides, inequality (1) cannot be extended to the full range of exponents $p$ with some finite strictly positive constant $c(p)$ unlike (4) and $(5)($ see $[8,4,6]$ and (49)).

\section{Proof of the main result}

\subsection{An anonymous Bellman function}

In this section we want to define a function $U: \mathbb{R}^{2} \rightarrow \mathbb{R}$ that satisfies some special properties. Let $\alpha \geq 2$ and let $\beta=\frac{\alpha}{\alpha-1} \leq 2$ be the conjugate exponent of $\alpha$. Let

$$
N_{\alpha}(x):={ }_{1} F_{1}\left(-\frac{\alpha}{2}, \frac{1}{2}, \frac{x^{2}}{2}\right)=\sum_{m=0}^{\infty} \frac{\left(-2 x^{2}\right)^{m}}{(2 m) !} \frac{\alpha}{2}\left(\frac{\alpha}{2}-1\right) \cdots\left(\frac{\alpha}{2}-m+1\right)=1-x^{2} \frac{\alpha}{2}+\ldots
$$

be the confluent hypergeometric function. $N_{\alpha}(x)$ satisfies the Hermite differential equation

$$
N_{\alpha}^{\prime \prime}(x)-x N_{\alpha}^{\prime}(x)+\alpha N_{\alpha}(x)=0 \quad \text { for } \quad x \in \mathbb{R}
$$

with initial conditions $N_{\alpha}(0)=1$ and $N_{\alpha}^{\prime}(0)=0$. Let $s_{\alpha}$ be the smallest positive zero of $N_{\alpha}$.

Set

$$
u_{\alpha}(x):= \begin{cases}-\frac{\alpha s_{\alpha}^{\alpha-1}}{N_{\alpha}^{\prime}\left(s_{\alpha}\right)} N_{\alpha}(x), & 0 \leq|x| \leq s_{\alpha} \\ s_{\alpha}^{\alpha}-|x|^{\alpha}, & s_{\alpha} \leq|x|\end{cases}
$$

Clearly $u_{\alpha}(x)$ is $C^{1}(\mathbb{R}) \cap C^{2}\left(\mathbb{R} \backslash\left\{s_{\alpha}\right\}\right)$ smooth even concave function. The concavity follows from Lemma A.1 and the fact that $N_{\alpha}^{\prime}\left(s_{\alpha}\right)<0$. Finally we define

$$
U(p, q):=|q|^{\alpha} u_{\alpha}\left(\frac{p}{|q|}\right) \quad \text { with } \quad U(p, 0)=-|p|^{\alpha} .
$$

For the first time the function $U(p, q)$ appeared in [8]. Later it was also used in [20, 21] in the form $\widetilde{u}(p, t)=U(p, \sqrt{t}), t \geq 0$. It was explained in [8] that $U(p, q)$ satisfies the following properties:

$$
\begin{aligned}
& U(p, q) \geq|q|^{\alpha} s_{\alpha}^{\alpha}-|p|^{\alpha} \quad \text { for all } \quad(p, q) \in \mathbb{R}^{2}, \quad \text { and when } q=0, \text { the equality holds; } \\
& 2 U(p, q) \geq U\left(p+a, \sqrt{a^{2}+q^{2}}\right)+U\left(p-a, \sqrt{a^{2}+q^{2}}\right) \text { for all } \quad(p, q, a) \in \mathbb{R}^{3} .
\end{aligned}
$$


We should refer to (9) as the obstacle condition, and to (10) as the main inequality. We caution the reader that in [8] one may not find (10) written explicitly but one will find its infinitesimal form

$$
\widetilde{u}_{t}+\frac{\widetilde{u}_{p p}}{2} \leq 0 \quad \text { for } \quad \widetilde{u}(p, t)=U(p, \sqrt{t}),
$$

which follows from the main inequality by expanding it into Taylor's series with respect to $a$ near $a=0$ and comparing the second order terms. Here $\widetilde{u}_{p p}$ is defined everywhere except the curve $|p / \sqrt{t}|=s_{\alpha}$ where $\widetilde{u}$ is only differentiable once.

In fact, the reverse implication also holds, i.e., one can derive (10) from (11) for this special $U$. This was done in the PhD thesis of Wang [21] but we will present a short proof in Section A.2, which partly follows the Davis argument. Essentially the same argument also appeared later in [1] in a slightly different setting.

The function $U(p, q)$ is essential in obtaining the result in the Davis paper, namely it is used in the proof of (4), and the argument goes as follows. First one shows that

$$
X_{t}=U\left(B_{t}, \sqrt{t}\right) \text { for } t \geq 0
$$

is a supermartingale which is guaranteed by (11). Finally, by the optional stopping theorem,

$$
\mathbb{E}\left(T^{\frac{\alpha}{2}} s_{\alpha}^{\alpha}-\left|B_{T}\right|^{\alpha}\right) \stackrel{(9)}{\leq} \mathbb{E} U\left(B_{T}, \sqrt{T}\right) \leq U(0,0)=0,
$$

which yields (4). One may notice that $U(p, q)$ is the minimal function with properties (9) and (10).

Davis mentions that the proof presented in his paper was suggested by an anonymous referee, and this explains the title of the current section.

\subsection{Dualizing the Bellman function $U(p, q)$ and going to the Hamming cube}

Set $\Psi(p, q, x, y):=p x+q y+U(p, q)$ for $x \in \mathbb{R}$ and $y \geq 0$. We define

$$
M(x, y)=\min _{q \leq 0} \sup _{p \in \mathbb{R}} \Psi(p, q, x, y) \quad \text { for } \quad x \in \mathbb{R}, y \geq 0 .
$$

Lemma 2.1. For each $(x, y) \in \mathbb{R} \times \mathbb{R}_{+}$, there exists $\left(p^{*}, q^{*}\right)=\left(p^{*}(x, y), q^{*}(x, y)\right)$ such that

$$
\min _{q \leq 0} \sup _{p \in \mathbb{R}} \Psi(p, q, x, y)=\max _{p \in \mathbb{R}} \inf _{q \leq 0} \Psi(p, q, x, y)=\Psi\left(p^{*}, q^{*}, x, y\right)
$$

and we have

$$
\Psi\left(p, q^{*}, x, y\right) \leq \Psi\left(p^{*}, q^{*}, x, y\right) \leq \Psi\left(p^{*}, q, x, y\right) \quad \text { for all } \quad(p, q) \in \mathbb{R} \times \mathbb{R}_{-} .
$$

Proof. First let us show that for each fixed $(x, y)$ the function $\Psi(p, q, x, y)$ is convex in $q$ and concave in $p$. The concavity in $p$ follows from Lemma A.1, and the fact that $U$ is even and $C^{1}$ smooth in $p$.

To verify the convexity in $q$, it is enough to show that the map $q \mapsto U(p, q)$ is convex for $|p| \leq|q| s_{\alpha}$. Set $z=\frac{|p|}{|q|} \in\left[0, s_{\alpha}\right]$. Then we have

$$
\begin{aligned}
& U_{q q}=|q|^{\alpha-2}\left[\alpha(\alpha-1) u_{\alpha}(z)-2(\alpha-1) z u_{\alpha}^{\prime}(z)+z^{2} u_{\alpha}^{\prime \prime}(z)\right] \stackrel{(7)}{=} \\
& |q|^{\alpha-2}\left[-(\alpha-1) z u_{\alpha}^{\prime}(z)+\left(z^{2}-\alpha+1\right) u_{\alpha}^{\prime \prime}(z)\right] .
\end{aligned}
$$

Since $u_{\alpha}(z)$ coincides with $N_{\alpha}(z)$ up to a positive constant, the convexity follows from Lemma A.1 and the fact that $\alpha \geq 2$. 
Notice that for each $(x, y) \in \mathbb{R} \times \mathbb{R}_{+}$the map

$$
(p, q) \mapsto p x+q y+|q|^{\alpha} u_{\alpha}\left(\frac{p}{|q|}\right)
$$

satisfies the assumptions of Theorem A.6 where we take $\left(p_{0}, q_{0}\right)=(0,0)$ (see Section A.3 in Appendix). Therefore the conclusions of Lemma 2.1 follow from Theorem A.6.

Lemma 2.2. For $\beta=\frac{\alpha}{\alpha-1}$, any $x, a, b \in \mathbb{R}$, and any $y \geq 0$ we have

$$
\begin{aligned}
& M(x, y) \geq\left(\frac{\alpha-1}{\alpha^{\beta}}\right)\left(|x|^{\beta}-\frac{y^{\beta}}{s_{\alpha}^{\beta}}\right) \text { and when } y=0 \text { the equality holds } \\
& 2 M(x, y) \geq M\left(x+a, \sqrt{a^{2}+(y+b)^{2}}\right)+M\left(x-a, \sqrt{a^{2}+(y-b)^{2}}\right) .
\end{aligned}
$$

The reader notices that dualization (12) produces inequality (17) that is different from (10).

Proof. Set

$$
\left(x_{ \pm}, y_{ \pm}\right):=\left(x \pm a, \sqrt{a^{2}+(y \pm b)^{2}}\right)
$$

Lemma 2.1 gives points $\left(p^{*}, q^{*}\right)$ and $\left(p^{ \pm}, q^{ \pm}\right)$corresponding to $(x, y)$ and $\left(x_{ \pm}, y_{ \pm}\right)$. It follows from (14) that to prove (17) it would be enough to find numbers $p \in \mathbb{R}$ and $q_{1}, q_{2} \leq 0$ such that

$$
2 \Psi\left(p, q^{*}, x, y\right) \geq \Psi\left(p^{+}, q_{1}, x_{+}, y_{+}\right)+\Psi\left(p^{-}, q_{2}, x_{-}, y_{-}\right) .
$$

The right choice will be

$$
p=\frac{p^{+}+p^{-}}{2} \text { and } q_{1}=q_{2}=-\sqrt{\left(\frac{p^{+}-p^{-}}{2}\right)^{2}+\left(q^{*}\right)^{2}},
$$

but let us explain it in details.

Notice that by Cauchy-Schwarz we have

$$
q_{1} \sqrt{a^{2}+(y+b)^{2}}+q_{2} \sqrt{a^{2}+(y-b)^{2}}-2 q^{*} y \leq-|a|\left(\sqrt{q_{1}^{2}-\left(q^{*}\right)^{2}}+\sqrt{q_{2}^{2}-\left(q^{*}\right)^{2}}\right)
$$

provided that $q_{1}, q_{2} \leq q^{*} \leq 0$. Indeed, we have

$$
\begin{aligned}
& q_{1} \sqrt{a^{2}+(y+b)^{2}}+q_{2} \sqrt{a^{2}+(y-b)^{2}}-2 q^{*} y= \\
& -\sqrt{\left(q_{1}^{2}-\left(q^{*}\right)^{2}\right)+\left(q^{*}\right)^{2}} \sqrt{a^{2}+(y+b)^{2}}-\sqrt{\left(q_{2}^{2}-\left(q^{*}\right)^{2}\right)+\left(q^{*}\right)^{2}} \sqrt{a^{2}+(y-b)^{2}}-2 q^{*} y \leq \\
& -|a| \sqrt{q_{1}^{2}-\left(q^{*}\right)^{2}}-\left|q^{*}(y+b)\right|-|a| \sqrt{q_{2}^{2}-\left(q^{*}\right)^{2}}-\left|q^{*}(y-b)\right|-2 q^{*} y \leq \\
& -|a|\left(\sqrt{q_{1}^{2}-\left(q^{*}\right)^{2}}+\sqrt{q_{2}^{2}-\left(q^{*}\right)^{2}}\right) .
\end{aligned}
$$

Denoting $r_{j}^{2}=q_{j}^{2}-\left(q^{*}\right)^{2}$ for $j=1,2$, we see that it is enough to find $p \in \mathbb{R}$ and $r_{1}, r_{2} \geq 0$ such that

$$
2\left(p x+U\left(p, q^{*}\right)\right) \geq-|a|\left(r_{1}+r_{2}\right)+p^{+} x_{+}+U\left(p^{+}, \sqrt{r_{1}^{2}+\left(q^{*}\right)^{2}}\right)+p^{-} x_{-}+U\left(p^{-}, \sqrt{r_{2}^{2}+\left(q^{*}\right)^{2}}\right) .
$$




\section{PaAta IVAnisvili, Fedor Nazarov, Alexander Volberg}

By choosing $p=\frac{p^{+}+p^{-}}{2}$, and substituting the values for $x_{ \pm}=x \pm a$ we see that it would suffice to find $r_{1}, r_{2} \geq 0$ such that

$$
2 U\left(\frac{p^{+}+p^{-}}{2}, q^{*}\right) \geq-|a|\left(r_{1}+r_{2}\right)+a\left(p^{+}-p^{-}\right)+U\left(p^{+}, \sqrt{r_{1}^{2}+\left(q^{*}\right)^{2}}\right)+U\left(p^{-}, \sqrt{r_{2}^{2}+\left(q^{*}\right)^{2}}\right) .
$$

We will choose $r_{1}=r_{2}=\frac{\left|p^{+}-p^{-}\right|}{2}$. It follows from $-|a|\left|p^{+}-p^{-}\right|+a\left(p^{+}-p^{-}\right) \leq 0$ that we only need to have the inequality

$$
2 U\left(\frac{p^{+}+p^{-}}{2}, q^{*}\right) \geq U\left(p^{+}, \sqrt{\left(\frac{p^{+}-p^{-}}{2}\right)^{2}+\left(q^{*}\right)^{2}}\right)+U\left(p^{-}, \sqrt{\left(\frac{p^{+}-p^{-}}{2}\right)^{2}+\left(q^{*}\right)^{2}}\right) .
$$

But this inequality follows from (10).

To verify the obstacle condition (16), notice that (9) for $U(p, q)$ gives

$$
M(x, y) \geq \min _{q \leq 0} \sup _{p}\left(p x+q y+|q|^{\alpha} s_{\alpha}^{\alpha}-|p|^{\alpha}\right)=\left(\frac{\alpha-1}{\alpha^{\beta}}\right)\left(|x|^{\beta}-\frac{y^{\beta}}{s_{\alpha}^{\beta}}\right) .
$$

Finally if $y=0$, then we obtain

$$
M(x, 0)=\max _{p} \inf _{q \leq 0}(p x+U(p, q)) \stackrel{(*)}{=} \max _{p}(p x+U(p, 0))=\sup _{p}\left(p x-|p|^{\alpha}\right)=\left(\frac{\alpha-1}{\alpha^{\beta}}\right)|x|^{\beta} .
$$

Equality $(*)$ follows from the fact that

$$
q \mapsto p x+U(p, q)
$$

is an even convex map.

Corollary 2.3. For any $a, x \in \mathbb{R}$, all $y, b \in \mathbb{R}^{N}$, and any $N \geq 1$, we have

$$
\frac{1}{2}\left(M\left(x+a, \sqrt{a^{2}+\|y+b\|^{2}}\right)+M\left(x-a, \sqrt{a^{2}+\|y-b\|^{2}}\right)\right) \leq M(x,\|y\|) .
$$

Proof. It follows from the definition of $M$ that the map $y \mapsto M(x, y)$ is decreasing in $y$ for $y \geq 0$. Therefore by (17) and the triangle inequality we obtain

$$
\begin{aligned}
& \frac{1}{2}\left(M\left(x+a, \sqrt{a^{2}+\|y+b\|^{2}}\right)+M\left(x-a, \sqrt{a^{2}+\|y-b\|^{2}}\right)\right) \leq \\
& M\left(x, \frac{\|y+b\|+\|y-b\|}{2}\right) \leq M(x,\|y\|) .
\end{aligned}
$$

The inequality (20) gives rise to the estimate

$$
\mathbb{E} M(f,|\nabla f|) \leq M(\mathbb{E} f, 0) \text { for all } f:\{-1,1\}^{n} \rightarrow \mathbb{R} .
$$

Indeed, the reader can find in [11] the passage from (20) to (21). In fact, inequality (20) is the same as

$$
\mathbb{E}_{x_{j}} M(f,|\nabla f|) \leq M\left(\mathbb{E}_{x_{j}} f,\left|\nabla \mathbb{E}_{x_{j}} f\right|\right) \text { for any } f:\{-1,1\}^{n} \rightarrow \mathbb{R},
$$


where $\mathbb{E}_{x_{j}}$ takes the average in the coordinate $x_{j}$, i.e.,

$$
\mathbb{E}_{x_{j}} f=\frac{1}{2}(f \underbrace{\left(x_{1}, \ldots, 1, \ldots, x_{n}\right)}_{\text {set } 1 \text { on the } j \text {-th place }}+f \underbrace{\left(x_{1}, \ldots,-1, \ldots, x_{n}\right)}_{\text {set }-1 \text { on the } j \text {-th place }}) .
$$

The rest follows by iterating (22), the fact that $\mathbb{E}=\mathbb{E}_{x_{1}} \ldots \mathbb{E}_{x_{n}}$ and $|\nabla \mathbb{E} f|=0$.

\subsection{The proof of Theorem 1.1}

We have

$$
\left(\frac{\alpha-1}{\alpha^{\beta}}\right) \mathbb{E}\left(|f|^{\beta}-\frac{|\nabla f|^{\beta}}{s_{\alpha}^{\beta}}\right) \stackrel{(16)}{\leq} \mathbb{E} M(f,|\nabla f|) \stackrel{(21)}{\leq} M(\mathbb{E} f, 0) \stackrel{(16)}{=}\left(\frac{\alpha-1}{\alpha^{\beta}}\right)|\mathbb{E} f|^{\beta}
$$

and this gives inequality (1).

\section{Remarks and Applications}

\subsection{Going from $U$ to $M$ : from Square function to the Hamming cube}

Let $g$ be an integrable function on $[0,1]$. Let $D([0,1])$ denote all dyadic intervals in $[0,1]$. Consider the dyadic martingale $g_{n}$ defined as follows

$$
g_{n}(x)=\sum_{|I|=2^{-n}, I \in D([0,1])}\langle g\rangle_{I} \mathbb{1}_{I}(x)
$$

where $\langle g\rangle_{I}=\frac{1}{|I|} \int_{I} g$. The square function $S(g)$ is defined as follows

$$
S(g)(x)=\left(\sum_{n=0}^{\infty}\left(g_{n+1}(x)-g_{n}(x)\right)^{2}\right)^{1 / 2} .
$$

For convenience we always assume that the number of nonzero terms in (23) is finite so that $S(g)(x)$ makes sense. Let $O(p, q)$ be a continuous real valued function, and suppose one wants to estimate the quantity $\int_{0}^{1} O(g, S(g))$ from above in terms of $\int_{0}^{1} g$. If one finds a function

$$
\begin{aligned}
& U(p, q) \geq O(p, q), \\
& 2 U(p, q) \geq U\left(p+a, \sqrt{a^{2}+q^{2}}\right)+U\left(p-a, \sqrt{a^{2}+q^{2}}\right),
\end{aligned}
$$

then one obtains (see [20]) the bound

$$
\int_{0}^{1} O(g, S(g)) \leq \int_{0}^{1} U(g, S(g)) \leq U\left(\int_{0}^{1} g, 0\right) .
$$

Conversely, suppose that the inequality

$$
\int_{0}^{1} O(g, S(g)) \leq F\left(\int_{0}^{1} g\right)
$$


holds for all integrable functions $g$ on $[0,1]$ and some $F$. Then there exists $U(p, q)$ such that the conditions (24), (25) are satisfied and $U(p, 0) \leq F(p)$. Indeed, consider the extremal problem

$$
U(p, q)=\sup _{g}\left\{\int_{0}^{1} O\left(g, \sqrt{S(g)^{2}+q^{2}}\right), \quad \int_{0}^{1} g=p\right\} .
$$

This $U$ satisfies (24) (take $g=p$ constant), and, in fact, it satisfies (25). The latter fact can be proved by using the standard Bellman principle (see Chapter 8, [17], and survey [16]). Besides,

$$
U(p, 0)=\sup _{g}\left\{\int_{0}^{1} O(g, S(g)), \quad \int_{0}^{1} g=p\right\} \leq F(p)
$$

because of (27). Therefore there is one to one correspondence between the extremal problems for the square function of the form (27) and the functions $U(p, q)$ with the properties (24) and (25).

The gradient estimates on the Hamming cube are more subtle. Take any real valued $\widetilde{O}(x, y)$ and suppose that we want to estimate $\mathbb{E} \widetilde{O}(f,|\nabla f|)$ from above in terms of $\mathbb{E} f$ for any $f:\{-1,1\}^{n} \rightarrow \mathbb{R}$ and for all $n \geq 1$. If one finds $M(x, y)$ such that

$$
\begin{aligned}
& M(x, y) \geq \widetilde{O}(x, y), \\
& 2 M(x, y) \geq M\left(x+a, \sqrt{a^{2}+(y+b)^{2}}\right)+M\left(x-a, \sqrt{a^{2}+(y-b)^{2}}\right),
\end{aligned}
$$

then ${ }^{1}$ one can obtain the estimate (see [11])

$$
\mathbb{E} \widetilde{O}(f,|\nabla f|) \leq \mathbb{E} M(f,|\nabla f|) \leq M(\mathbb{E} f, 0) .
$$

Thus finding such $M$ is sufficient to obtain the estimate but it is unclear whether conditions (28) and (29) are necessary to obtain the bound $\mathbb{E} \widetilde{O}(f,|\nabla f|) \leq M(\mathbb{E} f, 0)$. In other words we do not know what is the corresponding extremal problem for $M$, i.e., what is the right Bellman function $M$. The reason lies in the fact that there is an essential difference between the Hamming cube and the dyadic intervals, i.e., test functions do not concatenate in a good way on $\{-1,1\}^{n}$ as it happens for dyadic martingales.

Now we formulate an abstract theorem that formalizes our duality principle in a general setting.

Theorem 3.1. Let $I, J \subseteq \mathbb{R}$ be convex sets. Take an arbitrary $O(p, q) \in C\left(I \times \mathbb{R}_{+}\right)$, and let $U(p, q): I \times \mathbb{R}_{+} \rightarrow \mathbb{R}$ satisfy properties (24) and (25). Assume that for each $(x, y) \in J \times \mathbb{R}_{+}$, we have

$$
\min _{q \leq 0} \sup _{p \in I} p x+q y+U(p,|q|)=\max _{p \in I} \inf _{q \leq 0} p x+q y+U(p,|q|) .
$$

Then $M$ and $\widetilde{O}$ defined as

$$
\begin{aligned}
& M(x, y)=\min _{q \leq 0} \sup _{p \in I}(p x+q y+U(p,|q|)) \\
& \widetilde{O}(x, y)=\inf _{q \leq 0} \sup _{p \in I}(p x+q y+O(p,|q|))
\end{aligned}
$$

satisfy (29) and (28), and, thereby, (30) for any $f:\{-1,1\}^{n} \rightarrow J$ and any $n \geq 1$.

\footnotetext{
${ }^{1}$ We do also need to assume that $y \mapsto M(x, y)$ is decreasing in $y$ for each fixed $x$ to ensure Corollary 2.3. But if $M$ is $C^{1}$ smooth then $M_{y} \leq 0$ is guaranteed by (29). Indeed, if we take $a=0$ in (29) we obtain that $y \mapsto M(x, y)$ is concave for each $x$. Next, taking $y=b=0$ and sending $a \rightarrow 0+$, we obtain by Taylor's formula that $M_{y}(x, 0) \leq 0$. Therefore $M_{y}(x, y) \leq 0$.
} 
One may think that finding $U(p, q)$ with the property (25) is a difficult problem. Let us make a quick remark here that if it happens that $t \mapsto U(p, \sqrt{t})$ is convex for each fixed $p \in I$, then (25) is automatically implied by its infinitesimal form, i.e., by $U_{p p}+U_{q} / q \leq 0$ (see the proof of Lemma A.4).

Proof. The proof essentially repeats the proof of Lemma 2.2. Let us sketch the argument. Define $\Psi(p, q, x, y):=$ $p x+q y+U(p,|q|)$. The existence of a saddle point $\left(p^{*}, q^{*}\right)$ with properties (13) and (14) is guaranteed by Lemma A.5. The convexity of the set $I$ allows us to choose $p$ from $I$, and $q_{1}, q_{2} \in(-\infty, 0]$ according to (18). The rest of the proof of the theorem is the same as in Lemma 2.2. Inequality (32) follows from (24). Convexity of $J$ is needed, for example, to ensure that if $f:\{-1,1\}^{n} \rightarrow J$, then $\mathbb{E} f \in J$, so that (30) makes sense.

\subsection{Going from $M$ to $U$ : from Hamming cube to square function}

Another interesting observation is that equality (31) was lurking in a solution of a certain Monge-Ampère equation. For example, taking $a, b \rightarrow 0$ in (29), and using the Taylor's series expansion (assuming that $M$ is smooth enough) one obtains

$$
\left(\begin{array}{cc}
M_{x x}+\frac{M_{y}}{y} & M_{x y} \\
M_{x y} & M_{y y}
\end{array}\right) \leq 0 .
$$

When looking for the least function $M$ with $M \geq \widetilde{O}$ and (33), it is reasonable to assume that condition (33) should degenerate except, possibly, on the set where $M$ coincides with its obstacle $\widetilde{O}$. The degeneracy of (33) means that the determinant of the matrix in (33) is zero. This is a general Monge-Ampère type equation and, after an application of the exterior differential systems of Bryant-Griffiths (see [12]), we obtain that the solutions can be locally characterized as follows:

$$
\begin{aligned}
& x=-U_{p}(p, q), \\
& y=-U_{q}(p, q), \\
& M(x, y)=p x+q y+U(p, q),
\end{aligned}
$$

where $U$ satisfies the equation

$$
U_{p p}+\frac{U_{q}}{q}=0
$$

In [12] we used $u(p, t)=-U(p, \sqrt{2 t})$ instead of $U(p, q)$, in which case (35) becomes just the backward heat equation for $u(p, t)$. We will not formulate a formal statement but we do make a remark that such a reasoning allows us to guess the dual of $M$, i.e., to find $U$ given $M$. The way this guess works will be illustrated in Section 3.4 .

Our final remark is that one may try to use $U(p, q):=M(p, q)$ with $O(p, q):=\widetilde{O}(p, q)$ because (29) clearly implies (25). It will definitely give some estimate for the square function but not the sharp one. Indeed, for the sharp estimates, condition (25) for $U$ usually degenerates, namely (35) holds. On the other hand, if $M_{x x}+M_{y} / y=0$ and (33) holds, then $M_{x y}=0$, and

$$
M(x, y)=C\left(x^{2}-y^{2}\right)+D x+Q
$$

for some constants $C, D, Q \in \mathbb{R}$. This family of functions corresponds to the trivial inequality $\int_{0}^{1} S(g)^{2} \leq \int_{0}^{1} g^{2}$. Analogously, the best possible function $U$ satisfying (24) and (25) will almost never satisfy (33) except for a very particular case when $U(p, q)=C\left(p^{2}-q^{2}\right)+D p+Q$. 


\subsection{The dual to Log-Sobolev is Chang-Wilson-Wolff}

The function $M(x, y)=x \ln x-\frac{y^{2}}{2 x}$ satisfies (33) and, therefore, it gives the log-Sobolev inequality [12]. Its dual in the sense of (34) is $U(p, q)=e^{p-q^{2} / 2}$ (see Section 3.1.1 in [12] where $t=q^{2} / 2$ ). Notice that for this $U$, inequality (25) simplifies to

$$
2 e^{a^{2} / 2} \geq e^{a}+e^{-a}
$$

which is true since $(2 k) ! \geq 2^{k} k$ ! for $k \geq 0$. Therefore we obtain

Corollary 3.2. For any integrable $g$ on $[0,1]$, we have

$$
\int_{0}^{1} \exp \left(g-\frac{S^{2}(g)}{2}\right) \leq \exp \left(\int_{0}^{1} g\right) .
$$

This corollary immediately recovers the result of Chang-Wilson-Wolf [7] well-known to probabilists, namely for any $g$ with $\int_{0}^{1} g=0$ and $\|S(g)\|_{\infty}<\infty$, we have

$$
\int_{0}^{1} e^{g} \leq e^{\|S(g)\|_{\infty}^{2} / 2}
$$

Next, repeating a standard argument, namely, considering $t g$ and applying Chebyshev's inequality (see Theorem 3.1 in [7]), one obtains the superexponential bound

$$
\left|\left\{x \in[0,1]: g(x)-\int_{0}^{1} g \geq \lambda\right\}\right| \leq e^{-\frac{1}{2} \lambda^{2} /\|S g\|_{\infty}^{2}}
$$

for any $\lambda \geq 0$.

We should remind that the log-Sobolev inequality via the Herbst argument [13] gives Gaussian concentration inequalities, namely,

$$
\gamma\left(x \in \mathbb{R}^{n}: f(x)-\int_{\mathbb{R}^{n}} f d \gamma \geq \lambda\right) \leq e^{-\frac{1}{2} \lambda^{2} /\|\nabla f\|_{\infty}^{2}}
$$

for any $\lambda \geq 0$ and any smooth $f: \mathbb{R}^{n} \rightarrow \mathbb{R}$ with $\|\nabla f\|_{\infty}<\infty$. Here $\gamma$ is the standard Gaussian measure on $\mathbb{R}^{n}$.

In other words we just illustrated that estimates (39) and (38) are dual to each other in the sense of duality between functions $M=x \ln x-\frac{y^{2}}{2 x}$ and $U=e^{p-q^{2} / 2}$.

\subsection{Poincaré inequality 3/2: a simple proof via duality}

It was proved in [11] that for any $f:\{-1,1\}^{n} \rightarrow \mathbb{R}$, we have

$$
\mathbb{E} \Re(f+i|\nabla f|)^{3 / 2} \leq \mathfrak{R}(\mathbb{E} f)^{3 / 2},
$$

where $z^{3 / 2}$ is taken in the sense of the principal brunch in the upper half-plane. Inequality (40) improves Beckner's bound for a particular exponent [11]. Consider

$$
M(x, y)=\Re(x+i y)^{3 / 2}=\frac{1}{\sqrt{2}}\left(2 x-\sqrt{x^{2}+y^{2}}\right) \sqrt{\sqrt{x^{2}+y^{2}}+x} \text { for } \quad(x, y) \in \mathbb{R}^{2} .
$$

It was explained in [11] that to prove (40) it is enough to check that $M(x, y)$ satisfies (29), and the latter fact involved careful investigation of the roots of several very high degree polynomials with integer coefficients. Let us give a simple proof of (29) using our duality technique. 
Proposition 3.3. The function $M(x, y)=\mathfrak{R}(x+i y)^{3 / 2}$ satisfies (29) for all $x, a, b \in \mathbb{R}$ and $y \geq 0$.

Proof. $M(x, y)$ is a solution of the homogeneous Monge-Ampère equation (33), and therefore it has a representation of the form (34) (see Section 3.1.4 in [12]):

$$
\begin{aligned}
& x=-U_{p}(p, q) ; \\
& y=-U_{q}(p, q) \\
& U(p, q)=-\frac{4}{27}\left(p^{3}-3 p q^{2}\right) \\
& M(x, y)=p x+q y+U(p, q) .
\end{aligned}
$$

This leads us to the following guess

$$
\frac{1}{\sqrt{2}}\left(2 x-\sqrt{x^{2}+y^{2}}\right) \sqrt{\sqrt{x^{2}+y^{2}}+x}=\min _{q \leq 0} \sup _{p \geq 0}\left(x p+q y-\frac{4}{27}\left(p^{3}-3 p q^{2}\right)\right),
$$

which can be directly checked. Using Theorem A.6 with $\left(p_{0}, q_{0}\right)=(0,0)$, and following the proof of Lemma 2.2, it is enough to check that $U(p, q)$ satisfies (25). Notice that (25) is an identity for $U(p, q)=$ $-\frac{4}{27}\left(p^{3}-3 p q^{2}\right)$. This finishes the proof of the proposition.

\subsection{Sobolev inequalities}

\subsubsection{The Hamming cube $\{-1,1\}^{n}$}

For $p \in[1,2]$, let $c_{p}$ be the best possible constant such that

$$
c_{p}\left(\mathbb{E}|f|^{p}-|\mathbb{E} f|^{p}\right) \leq \mathbb{E}|\nabla f|^{p} \quad \text { for all functions } \quad f:\{-1,1\}^{n} \rightarrow \mathbb{R} .
$$

Our theorem implies that $c_{p} \geq s_{p^{\prime}}^{p}$ for $p \in(1,2]$. Notice that when $p=2$, we have $c_{2}=s_{2}^{2}=1$, and (41) recovers the classical Poincaré inequality. When $p \rightarrow 1+$ the constant $s_{p^{\prime}}^{p}$ tends to zero which should not be the case for $c_{p}$. Indeed, it follows from a deep result of Talagrand [19] that if $T_{p}$ is the best possible constant in the following estimate

$$
T_{p} \mathbb{E}|f-\mathbb{E} f|^{p} \leq \mathbb{E}|\nabla f|^{p} \quad \text { for all } \quad f:\{-1,1\}^{n} \rightarrow \mathbb{R},
$$

then $T_{p}>0$ for all $p \in[1, \infty)$. Now notice that $T_{1}=c_{1}, T_{2}=c_{2}$ and $T_{p} \geq c_{p}$ for $p \in(1,2)$. When $p>2$, by example (49), we must have $c_{p}=0$ unlike the fact that $T_{p}>0$ for $p>2$. So one may wonder whether the positivity of $T_{p}$ may not imply the positivity of $c_{p}$ on the interval $(1,2)$. Let us mention that this is not the case, in fact $2 c_{p} \geq T_{p}$ for $p \in(1,2)$. Indeed, it will suffice to prove that $2 \mathbb{E}|f-\mathbb{E} f|^{p} \geq \mathbb{E}|f|^{p}-|\mathbb{E} f|^{p}$. If $\mathbb{E} f=0$ this is obvious. Assume $\mathbb{E} f \neq 0$. Next, we show a simple inequality

$$
2|x-1|^{p}-|x|^{p}+1 \geq p(1-x) \text { for all } 1 \leq p \leq 2 \quad \text { and } \quad x \in \mathbb{R} .
$$

Plugging $x=f / \mathbb{E} f$, and taking the expectation, we obtain $2 \mathbb{E}|f-\mathbb{E} f|^{p} \geq \mathbb{E}|f|^{p}-|\mathbb{E} f|^{p}$. To verify (43), without loss of generality assume that $p>1$ (otherwise the inequality is trivial). Consider $g(x)=2|x-1|^{p}-$ $|x|^{p}+1$. Its second derivative changes signs at points $x$ which satisfy the equation $|x-1|=2^{1 /(2-p)}|x|$, i.e.,

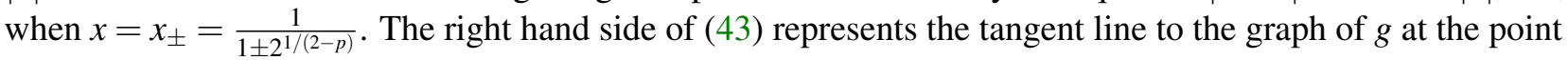
$x=1$. Clearly $g$ is convex on $\left[x_{+}, \infty\right)$. Therefore (43) is true on this interval. Next, $g$ is concave on $\left[x_{-}, x_{+}\right]$ and since $x_{-}<0$, we have $g \geq p(1-x)$ on $\left[0, x_{+}\right]$because $g(0)>p(1-0)$. Thus (43) is true for $x \geq 0$. For $x \leq 0$, by Bernoulli we have

$$
2|x-1|^{p}-|x|^{p}+1-p(1-x) \geq|x-1|^{p}+1-p(1-x) \geq 1-p x+1-p(1-x)=2-p \geq 0 .
$$


To the best of our knowledge, the constants $c_{p}, T_{p}$ are unknown for $p \in[1,2)$. There is a remarkable result of Ben-Efraim-Lust-Piquard [2] that $T_{p} \geq \frac{2}{\pi}$ for $1 \leq p \leq 2$.

This, combined to our theorem, gives the lower bound $T_{p} \geq \max \left\{\frac{2}{\pi}, s_{p^{\prime}}\right\}$ for $1 \leq p \leq 2$. However, due to the inequalities of Bobkov-Götze and Maurey-Pisier (see the next section), it is widely believed that $c_{1}=T_{1}=\sqrt{\frac{2}{\pi}}$.

An elegant idea of Naor-Schechtman [15] based on Burkholder's inequality [5] gives an estimate

$$
(p-1)^{p} \mathbb{E}|f-\mathbb{E} f|^{p} \leq \mathbb{E}_{x} \mathbb{E}_{x^{\prime}}\left|\sum_{j=1}^{n} x_{j}^{\prime} \partial_{j} f(x)\right|^{p} \quad 1<p \leq 2 .
$$

Let us show that our bound (2) obtained in Corollary 1.2 is better.

Proposition 3.4. For all $1<p<2$ we have

$$
s_{p^{\prime}}^{p} 2^{\frac{p-2}{2}} \min \left(1, \frac{\Gamma((p+1) / 2)}{\Gamma(3 / 2)}\right)>(p-1)^{p} \quad \text { for } \quad 1<p<2 .
$$

Proof. We estimate $s_{p^{\prime}}$ from below by $\sqrt{2 / p^{\prime}}$ (see Lemma A.2). Using $\Gamma(3 / 2)=\sqrt{\pi} / 2$ we see that to prove (44) it is enough to show the following two inequalities

$$
\begin{aligned}
& \left(\frac{2}{p^{\prime}}\right)^{p / 2} 2^{\frac{p-2}{2}}>(p-1)^{p} \quad \text { for } 1<p<2 ; \\
& \left(\frac{2}{p^{\prime}}\right)^{p / 2} 2^{\frac{p}{2}} \frac{\Gamma((p+1) / 2)}{\sqrt{\pi}}>(p-1)^{p} \quad \text { for } \quad p_{0}<p<2,
\end{aligned}
$$

where $p_{0} \approx 1.847 \ldots$ is the solution of the equation $\Gamma((p+1) / 2)=\sqrt{\pi} / 2$ on the interval $(1,2)$. Inequality (45) simplifies to $2^{2-\frac{2}{p}}>p(p-1)$ which is true because the left hand side is concave, and the right hand side is convex on $[1,2]$. To show (46) it is enough to verify that

$$
4\left(\frac{\Gamma((p+1) / 2)}{\sqrt{\pi}}\right)^{2 / p}>p(p-1) \text { for } 1<p<2 .
$$

The latter inequality we rewrite as follows

$$
\frac{p \ln (4)-\ln (\pi)}{2}+\ln \left(\Gamma\left(\frac{p+1}{2}\right)\right)-\frac{p \ln (p(p-1))}{2}>0 .
$$

Since the Trigamma function is convex

$$
(\ln \Gamma(z))^{\prime \prime}=\sum_{n=0}^{\infty} \frac{1}{(z+n)^{2}},
$$

we estimate $\ln (\Gamma((p+1) / 2))$ from below by its tangent line at point $p=2$, i.e.,

$$
\ln \left(\Gamma\left(\frac{p+1}{2}\right)\right)>\ln (\sqrt{\pi} / 2)+(1-\gamma / 2-\ln (2))(p-2) \quad \text { for } \quad p_{0}<p<2 \text {, }
$$

here $\gamma$ is Euler's constant. It is enough to show that

$$
\frac{p \ln (4)-\ln (\pi)}{2}+\ln (\sqrt{\pi} / 2)+(1-\gamma / 2-\ln (2))(p-2)-\frac{p \ln (p(p-1))}{2}>0 .
$$

The left hand side is concave on the interval $(1+\sqrt{2} / 2,2)$, and at the endpoint cases we have the inequality. Notice that $(1+\sqrt{2} / 2)=1.7 \ldots<p_{0}=1.82 \ldots$, and this finishes the proof. 


\subsubsection{Gaussian measure on $\mathbb{R}^{n}$}

The application of the Central Limit Theorem to (1) gives a dimension independent Sobolev inequality.

Corollary 3.5. For any smooth bounded $f: \mathbb{R}^{n} \rightarrow \mathbb{R}$ and any $n \geq 1$, we have

$$
s_{p^{\prime}}^{p}\left(\int_{\mathbb{R}^{n}}|f|^{p} d \gamma-\left|\int_{\mathbb{R}^{n}} f d \gamma\right|^{p}\right) \leq \int_{\mathbb{R}^{n}}|\nabla f|^{p} d \gamma .
$$

The best possible constant in (47), unlike $s_{p^{\prime}}^{p}$, should not degenerate when $p \rightarrow 1+$. Indeed, (see [14], pp. 115) one has

$$
\sqrt{\frac{2}{\pi}} \int_{\mathbb{R}^{n}}\left|f-\int_{\mathbb{R}^{n}} f d \gamma\right| d \gamma \leq \int_{\mathbb{R}^{n}}|\nabla f| d \gamma
$$

where the constant $\sqrt{\frac{2}{\pi}}$ is the best possible in the left hand side of (48). We should mention that estimate (48) can be also easily obtained by a remarkable trick of Maurey-Pisier [18].

Notice that (47) cannot be extended for the range of exponents $p>2$ with some positive constant $C(p)$ instead of $s_{p^{\prime}}^{p}$. Indeed, assume the contrary. Consider $n=1$ and take $f(x)=1+a x$. Using Jensen's inequality, we obtain

$$
\left(1+a^{2}\right)^{p / 2}=\left(\int_{\mathbb{R}}|1+a x|^{2} d \gamma\right)^{p / 2} \leq \int_{\mathbb{R}}|1+a x|^{p} d \gamma \stackrel{(47)}{\leq} \frac{|a|^{p}}{C(p)}+1 .
$$

Therefore, taking $a \rightarrow 0$, we obtain the contradiction with $p a^{2} / 2>\frac{|a|^{p}}{C(p)}$ for $p>2$.

\subsection{Discrete surface measure}

Let $A \subset\{-1,1\}^{n}$ be a subset of the Hamming cube with cardinality $|A|=2^{n-1}$. Define $w_{A}:\{-1,1\}^{n} \rightarrow \mathbb{N} \cup\{0\}$ so that $w_{A}(x)$ is the number of boundary edges to $A$ containing $x$, i.e., $w_{A}(x)$ counts the number of edges with one endpoint in $A$ and another one in the complement of $A$ such that one of the endpoints is $x$. Clearly $w_{A}(x)=0$ if $x$ is in the "strict interior" of $A$, or in the "strict complement" of $A$, and it is nonzero if and only if $x$ is on the "boundary" of $A$. Notice that $w_{A}(x)$ can be nonzero for some $x \notin A$. The function $w_{A}$ maybe be understood as a discrete surface measure of the boundary of $A$. Consider the following quantity

$$
\sigma(p)=\inf _{A \subset\{-1,1\}^{n},|A|=2^{n-1}} \mathbb{E} w_{A}^{p / 2}(x) .
$$

It follows from Harper's edge-isoperimetric inequality [10] that $\sigma(2)=1$ and the value is attained on the halfcube. The monotonicity of $\sigma(p)$ in $p$ implies that $\sigma(p)=1$ for all $p \geq 2$. Also notice that considering Hamming balls, one can easily show that $\sigma(p)=0$ for $0 \leq p<1$. Therefore the first nontrivial value is $\sigma(1)$. In this case it follows from Bobkov's inequality (see [3] and references therein) that $\sigma(1) \geq \sqrt{\frac{2}{\pi}} \approx 0.79$, and by monotonicity we obtain that $\sigma(p) \geq \sqrt{\frac{2}{\pi}}$ which is definitely not sharp when $p \rightarrow 2-$.

Define $f:\{-1,1\}^{n} \rightarrow\{-1,1\}$ as follows: $f(x)=1$ if $x \in A$ and $f(x)=-1$ if $x \notin A$. Clearly $|\nabla f(x)|^{2}=$ $w_{A}(x)$. Applying (1) to $f$, we obtain

$$
\mathbb{E} w_{A}^{p / 2}(x) \geq s_{p^{\prime}}^{p}
$$

Inequality (51) gives the lower bound $\sigma(p) \geq s_{p^{\prime}}^{p}$ which tends to 1 as $p \rightarrow 2-$, but fails to be sharp when $p \rightarrow 1+$. Thus combining this result with Bobkov's inequality we obtain the bound

$$
1 \geq \sigma(p) \geq \max \left\{\sqrt{\frac{2}{\pi}}, s_{p^{\prime}}^{p}\right\} \quad \text { for } \quad 1 \leq p \leq 2 .
$$




\section{A Appendix}

\section{A.1 Properties of $N_{\alpha}(t)$}

Lemma A.1. For any $\alpha \geq 2$, we have $0<s_{\alpha} \leq 1$. In addition $s_{\alpha}$ is decreasing in $\alpha>0$, and $N_{\alpha}^{\prime}(t), N_{\alpha}^{\prime \prime}(t) \leq 0$ on $\left[0, s_{\alpha}\right]$ for $\alpha>0$.

Proof. Consider $G_{\alpha}(t):=e^{-t^{2} / 4} N_{\alpha}(t)$. Notice that the zeros of $G_{\alpha}$ and $N_{\alpha}$ are the same. It follows from (7) that

$$
G_{\alpha}^{\prime \prime}+\left(\alpha+\frac{1}{2}-\frac{t^{2}}{4}\right) G_{\alpha}=0, \quad G_{\alpha}(0)=1 \quad \text { and } \quad G_{\alpha}^{\prime}(0)=0 .
$$

Besides we know that the solution is even. Consider the critical case $\alpha=2$. In this case $G_{2}(t)=e^{-t^{2} / 4}\left(1-t^{2}\right)$ and the smallest positive zero is $s_{2}=1$. Therefore it follows from the Sturm comparison principle that $0<s_{\alpha}<1$ for $\alpha>2$ (see below). Moreover, the same principle applied to $G_{\alpha_{1}}$ and $G_{\alpha_{2}}$ with $\alpha_{1}>\alpha_{2}$ implies that $G_{\alpha_{1}}$ has a zero inside the interval $\left(-s_{\alpha_{2}}, s_{\alpha_{2}}\right)$. Thus we conclude that $s_{\alpha}$ is decreasing in $\alpha$.

To verify that $N_{\alpha}^{\prime}, N_{\alpha}^{\prime \prime} \leq 0$ on $\left[0, s_{\alpha}\right]$, first we claim that

$$
N_{\alpha_{2}} \geq N_{\alpha_{1}} \quad \text { on } \quad\left[0, s_{\alpha_{1}}\right]
$$

for $\alpha_{1}>\alpha_{2}>0$. Indeed the proof works in the same way as the proof of Sturm's comparison principle. For the convenience of the reader we decided to include the argument. As before, consider $G_{\alpha_{j}}=e^{-t^{2} / 4} N_{\alpha_{j}}$. It is enough to show that $G_{\alpha_{2}} \geq G_{\alpha_{1}}$ on $\left[0, s_{\alpha_{1}}\right]$. It follows from (53) that $G_{\alpha_{2}}^{\prime \prime}(0)>G_{\alpha_{1}}^{\prime \prime}(0)$. Therefore, using the Taylor series expansion at the point 0 , we see that the claim is true at some neighbourhood of zero, say $[0, \varepsilon)$ with $\varepsilon$ sufficiently small. Next we assume the contrary, i.e., that there is a point $a \in\left[\varepsilon, s_{\alpha_{1}}\right]$ such that $G_{\alpha_{2}} \geq G_{\alpha_{1}}$ on $[0, a], G_{\alpha_{2}}(a)=G_{\alpha_{1}}(a)$ and $G_{\alpha_{2}}^{\prime}(a)<G_{\alpha_{1}}^{\prime}(a)$ (notice that the case $G_{\alpha_{2}}^{\prime}(a)=G_{\alpha_{1}}^{\prime}(a)$, by the uniqueness theorem for ODEs, would imply that $G_{\alpha_{2}}=G_{\alpha_{1}}$ everywhere, which is impossible). Consider the Wronskian

$$
W=G_{\alpha_{1}}^{\prime} G_{\alpha_{2}}-G_{\alpha_{1}} G_{\alpha_{2}}^{\prime} .
$$

We have $W(0)=0$ and $W(a)=G_{\alpha_{1}}(a)\left(G_{\alpha_{1}}^{\prime}(a)-G_{\alpha_{2}}^{\prime}(a)\right) \geq 0$. On the other hand, we have

$$
W^{\prime}=\left(\alpha_{2}-\alpha_{1}\right) G_{\alpha_{1}} G_{\alpha_{2}}<0 \quad \text { on } \quad[0, a),
$$

which is a clear contradiction, and this proves the claim.

It follows from (6) that

$$
N_{\alpha}^{\prime \prime}=-\alpha N_{\alpha-2}
$$

and inequalities $N_{\alpha-2} \geq N_{\alpha} \geq 0$ on $\left[0, s_{\alpha}\right]$ imply that $N_{\alpha}^{\prime \prime} \leq 0$ on $\left[0, s_{\alpha}\right]$. Since $N_{\alpha}^{\prime}(0)=0$ and $N_{\alpha}^{\prime \prime} \leq 0$ on $\left[0, s_{\alpha}\right]$, we must have $N_{\alpha}^{\prime} \leq 0$ on $\left[0, s_{\alpha}\right]$.

Lemma A.2. We have $s_{\alpha} \geq \sqrt{\frac{2}{\alpha}}$ for all $\alpha \geq 2$.

Proof. Notice that $G_{2}(x)=e^{-x^{2} / 4}\left(1-x^{2}\right)$ satisfies (53) with $\alpha=2$. Now consider $V_{\alpha}(x):=G_{2}(x \sqrt{\alpha / 2})$. The function $V_{\alpha}(x)$ satisfies the equation

$$
V_{\alpha}^{\prime \prime}(x)+\left(\alpha+\frac{\alpha}{4}-\frac{\alpha^{2} x^{2}}{16}\right) V_{\alpha}(x)=0
$$

and $V_{\alpha}(0)=1, V_{\alpha}^{\prime}(0)=0$. Notice that $V_{\alpha}(x)>0$ on $[0, \sqrt{2 / \alpha})$. Since

$$
\alpha+\frac{\alpha}{4}-\frac{\alpha^{2} x^{2}}{16} \geq \alpha+\frac{1}{2}-\frac{x^{2}}{4} \quad \text { for } \quad x \in[0, \sqrt{2 / \alpha}),
$$

it follows from the Sturm comparison principle (see the previous discussions) that $G_{\alpha}>V_{\alpha}>0$ on $(0, \sqrt{2 / \alpha})$. Thus we obtain that $s_{\alpha} \geq \sqrt{2 / \alpha}$. 


\section{A.2 Heat inequality}

Let $U(p, q)$ be defined as in (8).

Lemma A.3. For any $p \in \mathbb{R}$, the map

$$
t \mapsto U(p, \sqrt{t}) \quad \text { for } \quad t \geq 0
$$

is convex.

Proof. Without loss of generality, assume that $p \geq 0$. We recall that $U(p, \sqrt{t})=t^{\alpha / 2} u_{\alpha}(p / \sqrt{t})$. Since $\alpha \geq 2$, the only interesting case to consider is when $p / \sqrt{t}<s_{\alpha}$ (otherwise $t^{\alpha / 2}$ is convex). In this case we have $U(p, \sqrt{t})=t^{\alpha / 2} N_{\alpha}(p / \sqrt{t})$ up to a positive constant which we are going to ignore, and, therefore, by (7) we have $(U(p, \sqrt{t}))_{t}+\frac{(U(p, \sqrt{t}))_{p p}}{2}=0$. Using (54), we obtain

$$
(U(p, \sqrt{t}))_{t}=-\frac{(U(p, \sqrt{t}))_{p p}}{2}=-\frac{1}{2} t^{\frac{\alpha}{2}-1} N_{\alpha}^{\prime \prime}(p / \sqrt{t})=\frac{\alpha}{2} t^{\frac{\alpha-2}{2}} N_{\alpha-2}(p / \sqrt{t}) .
$$

Therefore it would be enough to show that for any $\gamma \geq 0$, the function $\frac{N_{\gamma}(x)}{x^{\gamma}}$ is decreasing for $x \in\left(0, s_{\gamma+2}\right)$. Differentiating, and using (7) again, we obtain

$$
\frac{\mathrm{d}}{\mathrm{d} x}\left(\frac{N_{\gamma}(x)}{x^{\gamma}}\right)=\frac{N_{\gamma}^{\prime \prime}(x)}{x^{\gamma+1}}
$$

which is nonpositive by Lemma A.1.

The next lemma, together with Lemma A.3 and (11), implies that $U(p, q)$ satisfies (10).

Lemma A.4 (Barthe-Mauery [1]). Let $J$ be a convex subset of $\mathbb{R}$, and let $V(p, q): J \times \mathbb{R}_{+} \rightarrow \mathbb{R}$ be such that

$$
\begin{aligned}
& V_{p p}+\frac{V_{q}}{q} \leq 0 \quad \text { for all } \quad(p, q) \in J \times \mathbb{R}_{+} ; \\
& t \mapsto V(p, \sqrt{t}) \quad \text { is convex for each fixed } \quad p \in J .
\end{aligned}
$$

Then for all $(p, q, a)$ with $p \pm a \in J$ and $q \geq 0$, we have

$$
2 V(p, q) \geq V\left(p+a, \sqrt{a^{2}+q^{2}}\right)+V\left(p-a, \sqrt{a^{2}+q^{2}}\right) .
$$

The lemma says that the global discrete inequality (58) is in fact implied by its infinitesimal form (56) under the extra condition (57).

Proof. The argument is borrowed from [1]. The similar argument was used by Davis [8] in obtaining sharp square function estimates from the ones for the Brownian motion.

Without loss of generality assume $a \geq 0$. Consider the process

$$
X_{t}=V\left(p+B_{t}, \sqrt{q^{2}+t}\right), \quad t \geq 0 .
$$

Here $B_{t}$ is the standard Brownian motion starting at zero. It follows from Ito's formula together with (56) that $X_{t}$ is a supermartingale. Let $\tau$ be the stopping time

$$
\tau=\inf \left\{t \geq 0: B_{t} \notin(-a, a)\right\} .
$$


It follows from the optional stopping theorem that

$$
\begin{aligned}
& V(p, q)=X_{0} \geq \mathbb{E} X_{\tau}=\mathbb{E} V\left(p+B_{\tau}, \sqrt{q^{2}+\tau}\right)= \\
& P\left(B_{\tau}=-a\right) \mathbb{E}\left(V\left(p-a, \sqrt{q^{2}+\tau}\right) \mid B_{\tau}=-a\right)+P\left(B_{\tau}=a\right) \mathbb{E}\left(V\left(p+a, \sqrt{q^{2}+\tau}\right) \mid B_{\tau}=a\right)= \\
& \frac{1}{2}\left(\mathbb{E}\left(V\left(p-a, \sqrt{q^{2}+\tau}\right) \mid B_{\tau}=-a\right)+\mathbb{E}\left(V\left(p+a, \sqrt{q^{2}+\tau}\right) \mid B_{\tau}=a\right)\right) \geq \\
& \frac{1}{2}\left(V\left(p-a, \sqrt{q^{2}+\mathbb{E}\left(\tau \mid B_{\tau}=-a\right)}\right)+V\left(p+a, \sqrt{q^{2}+\mathbb{E}\left(\tau \mid B_{\tau}=a\right)}\right)\right)= \\
& \frac{1}{2}\left(V\left(p-a, \sqrt{q^{2}+a^{2}}\right)+V\left(p+a, \sqrt{q^{2}+a^{2}}\right)\right) .
\end{aligned}
$$

Notice that we have used $P\left(B_{\tau}=a\right)=P\left(B_{\tau}=-a\right)=1 / 2, \mathbb{E}\left(\tau \mid B_{\tau}=a\right)=\mathbb{E}\left(\tau \mid B_{\tau}=-a\right)=a^{2}$, and the fact that the map $t \mapsto V(p, \sqrt{t})$ is convex together with Jensen's inequality.

\section{A.3 Minimax theorem for noncompact sets}

Let $P, Q$ be nonempty closed convex sets in $\mathbb{R}$. We say that a pair $\left(p^{*}, q^{*}\right) \in P \times Q$ is a saddle point of $f$ on $P \times Q$ if

$$
f\left(p, q^{*}\right) \leq f\left(p^{*}, q^{*}\right) \leq f\left(p^{*}, q\right) \text { for all }(p, q) \in P \times Q .
$$

Lemma A.5. The function $f$ defined on $P \times Q$ with real values possesses a saddle point $\left(p^{*}, q^{*}\right)$ on $P \times Q$ if and only if

$$
\max _{p \in P} \inf _{q \in Q} f(p, q)=\min _{q \in Q} \sup _{p \in P} f(p, q),
$$

and this number is then equal to $f\left(p^{*}, q^{*}\right)$.

For the proof we refer the reader to Proposition 1.2 in [9], pp. 167.

Theorem A.6. Suppose that $f: P \times Q \rightarrow \mathbb{R}$ is continuous, concave in $p$, convex in $q$, and there exists $\left(p_{0}, q_{0}\right) \in P \times Q$ such that

$$
\lim _{p \in P,|p| \rightarrow \infty} f\left(p, q_{0}\right)=-\infty \quad \text { and } \lim _{q \in Q,|q| \rightarrow \infty} f\left(p_{0}, q\right)=+\infty .
$$

Then $f$ possesses at least one saddle point on $P \times Q$ and

$$
f\left(p^{*}, q^{*}\right)=\min _{q \in Q} \sup _{p \in P} f(p, q)=\max _{p \in P} \inf _{q \in Q} f(p, q) .
$$

The theorem is Proposition 2.2 in [9], pp. 173.

\section{Acknowledgments}

We are very grateful to several people for discussions and suggestions that led us to noticing the duality between the Hamming cube and the square function: G. Aubrun for valuable remarks on optimizers in (50); D. Bilyk for providing the reference to sharp constants for Square functions [20]; R. O'Donell for providing the references; R. Latała for pointing out example (49); S. Petermichl for bringing our attention to Bellman functions in Square function estimates and Poincaré inequalities for the Gaussian measure; S. Treil for attracting our attention to Chang-Wilson-Wolff's superexponential bound (Corollary 38) and its similarity to the Gaussian concentration inequality; R. van Handel for references, including (3) and (48), and making several important remarks. We thank an anonymous referee for helpful comments and remarks. 
SQUARE FUnCTIONS AND THE HAMMING CUBE: DUALITY

\section{References}

[1] F. Barthe, B. Maurey, Some remarks on isoperimetry of Gaussian type, Annales de l'Institut Henri Poincare (B) Probability and Statistics, Vol. 36, Iss. 4, pp. 419-434 4, 15

[2] L. Ben-Efraim, F. Lust-Piquard, Poincaré type inequalities on the discrete cube and in the CAR algebra, Probability Theory and Related Fields, Vol. 141, Iss. 3-4, pp. 569-602 (2008) 2, 12

[3] S. G. Bob Kov, F. GöTze, Discrete isoperimetric and Poincaré-type inequalities, Probab. Theory Relat. Fields 114, 245-277 (1999) 13

[4] D. Burkholder, Sharp inequalities for martingales and stochastic integrals, Colloque Paul Lévy (Palaiseau, 1987), Ast'erisque 157-158 (1988), 75-94 3

[5] D. L. BurKholder, Boundary Value Problems and Sharp Inequalities for Martingale Transforms, Ann. Probab., Vol. 12, No. 3, (1984), 647-702 12

[6] D. L. BuRKholder, R. F. Gundy, Extrapolation and interpolation of quasi-linear operators on martingales, Acta. Math., Vol. 124 (1970), 249-304 3

[7] A. Chang, J. M. Wilson, TH. WolfF, Some weighted norm inequalities concerning the Schrödinger operators, Comment. Math. Helvetici, Vol. 60, 1985, 217-246 10

[8] B. DAVIS, On the $L^{p}$ norms of stochastic integrals and other martingales, Duke Math. J. Vol. 43, pp. 697-704 (1976) 3, 4, 15

[9] I. Ekelan, R. Temam, Convex analysis and variational problems, North-Holland Publishing Company, Amsterdam, 197616

[10] L. H. HARPER, Optimal numberings and isoperimetric problems on graphs, J. Combin. Theory, pp. 385-393, 199613

[11] P. IVanisvili, A. Volberg, Poincaré inequality 3/2 on the Hamming cube, preprint arXiv:1608.4021 $3,6,8,10$

[12] P. IVANis Vili, A. Volberg, Isoperimetric functional inequalities via the maximum principle: the exterior differential systems approach, preprint arXiv: 1511.06895 9, 10, 11

[13] M. Ledoux, Concentration of measure and logarithmic Sobolev inequalities, In Séminare de Probabilités XXXIII, 120-216. Lecture Notes in Math. Vol. 1709. Springer, Berlin, 199910

[14] M. Ledoux, Isoperimetry and Gaussian Analysis, Lectures on Probability Theory and Statistics, Vol. 1648 of the series Lecture Notes in Mathematics pp 165-294 13

[15] A. Naor G. Schechtman, Remarks on Non linear Type and Pisier's Inequality, Journal für die reine und angevandte Mathematik (Crelle's Journal) 552 (2002) 213-236 2, 12

[16] F. Nazarov, S. Treil, A. Volberg, Bellman function in stochastic control and harmonic analysis, Systems, approximation, singular integral operators, and related topics (Bordeaux, 2000), 393-423, Oper. Theory Adv. Appl., 129, Birkhäuser, Basel, 20018

[17] A. OseKowski, Sharp Martingale and Semimartingale Inequalities, Monografie Matematyczne Vo. 72, Springer, Basel 8 
[18] G. PISIER, Probabilistic methods in the geometry of Banach spaces, in "Probability and Analysis, Varenna (Italy) 1985”, Lecture Notes in Math. 1206, 167-241, Springer Verlag (1986) 13

[19] M. TAlagRAnd, Isoperimetry, logarithmic Sobolev inequalities on the discrete cube, and Margulis' graph connectivity, Geom. Funct. Anal. 3, No. 3, 295-314 (1993) 11

[20] G. WANG, Sharp square function inequalities for conditionally symmetric martingales, Transaction of the American Mathematical Society, Vol. 328, No. 1 (1991) 3, 7, 16

[21] G. WANG, Some sharp inequalities for conditionally symmetric martingales, Ph.D. Thesis, University of Illinois at Urbana-Champaign, 1989 3, 4

\title{
AUTHORS
}

Paata Ivanisvili

Mathematics Department

Princeton University

Princeton, NJ 08544

and

Department of Mathematics

University of California, Irvine

Irvine, CA 92697-3875

paatai [at] princeton [dot] edu

Fedor Nazarov

Department of Mathematics

Kent State University

Kent, OH 44240

nazarov [at] math [dot] kent [dot] edu

\author{
Alexander Volberg \\ Department of Mathematics \\ Michigan State University \\ East Lansing, MI 48823 \\ volberg [at] math [dot] msu [dot] edu
}

